NIST Advanced Manufacturing Series 100-35

\title{
Thermal Calibration of Commercial Melt Pool Monitoring Sensors on a Laser Powder Bed Fusion System
}

Brandon Lane

Lars Jacquemetton

Martin Piltch

Darren Beckett

This publication is available free of charge from:

https://doi.org/10.6028/NIST.AMS.100-35

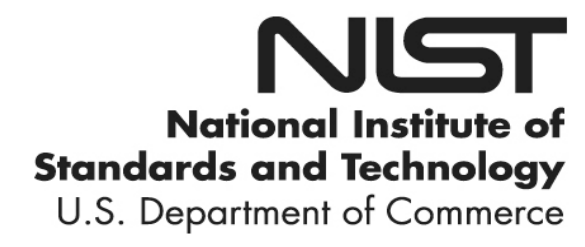




\title{
NIST Advanced Manufacturing Series 100-35
}

\section{Thermal Calibration of Commercial Melt Pool Monitoring Sensors on a Laser Powder Bed Fusion System}

\author{
Brandon Lane \\ Intelligent Systems Division \\ Engineering Laboratory
}

Lars Jacquemetton

Martin Piltch

Darren Beckett

Sigma Labs, Inc.

Santa Fe, New Mexico

This publication is available free of charge from:

https://doi.org/10.6028/NIST.AMS.100-35

July 2020

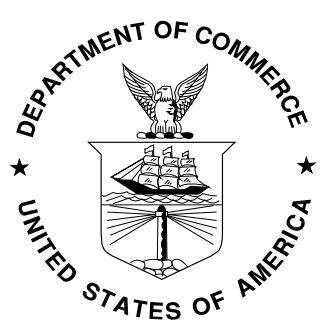

U.S. Department of Commerce Wilbur L. Ross, Jr., Secretary

National Institute of Standards and Technology Walter Copan, NIST Director and Undersecretary of Commerce for Standards and Technology 
Certain commercial entities, equipment, or materials may be identified in this document in order to describe an experimental procedure or concept adequately. Such identification is not intended to imply recommendation or endorsement by the National Institute of Standards and Technology, nor is it intended to imply that the entities, materials, or equipment are necessarily the best available for the purpose.

National Institute of Standards and Technology Advanced Manufacturing Series 100-35

Natl. Inst. Stand. Technol. Adv. Man. Ser. 100-35, 20 pages (July 2020)

This publication is available free of charge from: https://doi.org/10.6028/NIST.AMS.100-35 


\begin{abstract}
Co-axial melt pool monitoring in laser powder bed fusion (LPBF) additive manufacturing (AM) often utilizes various photodetectors to acquire signals relatable to the dynamic thermal melt pool phenomena. In turn, it is expected that these thermal signatures are relatable to the quality of the fabrication and can therefore the final AM part. To relate these signal values to real, physical temperatures, a thermal calibration must be done. However, the relatively high temperatures and small size of typical LPBF melt pools cannot be easily replicated by most thermal calibration sources. This paper describes a potential method for thermal calibration, which uses a lower temperature, commercial off-the-shelf calibration blackbody. The method calculates an 'effective' temperature for a hypothetical small source of any diameter, which would have the same radiance temperature of the larger, lower temperature blackbody. The paper details the theoretical rationale, provides a proof-of-concept calculation, then demonstrates the procedures conducted on a commercial LPBF melt pool monitoring system. Finally, while this method itself does not provide an absolute calibration nor ability to ascribe real melt pool temperatures to the melt pool monitoring sensor signals, discussion is provided that details the practical utility, details why the measured calibration values are realistic, and describes future improvements to the methodology.
\end{abstract}

\title{
Keywords
}

Laser powder bed fusion; additive manufacturing; process monitoring; temperature calibration; 


\section{Table of Contents}

1. Introduction ..............................................................................................................................

2. 'Equivalent Source' Theory ............................................................................................5

3. Measurement Setup ........................................................................................................

4. Calibration Results ...................................................................................................11

5. Discussion ..............................................................................................................................14

6. Improvements and Future Work ..........................................................................................15

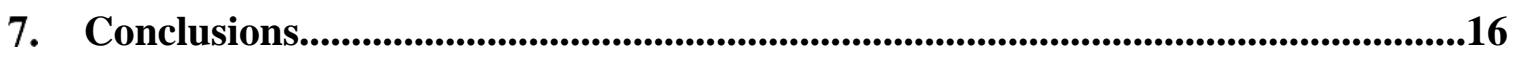

References..................................................................................................................................17

\section{List of Figures}

Figure 1: Schematic of optical layout of PrintRite3D co-axial melt pool monitoring system showing TEPTM \& TED ${ }^{\mathrm{TM}}$ sensors.

Figure 2: Left. Calculated temperature-dependent radiant flux from two sources at $10 \mathrm{~mm}$ diameter $\left(A_{b b}\right)$ and $200 \mu \mathrm{m}$ diameter $\left(A_{m p}\right)$. Right: Graphical depiction of the relative areas of a $10 \mathrm{~mm}$ and $200 \mu \mathrm{m}$ diameter source.

Figure 3: Measurement of the sensor spatial response function $S_{n}(x, y)$. (a) Experiment setup, consisting of a mirrored aperture (100 $\mu \mathrm{m}$ diameter), back-lit by fiber light and mounted on orthogonal micrometer stages. (b) Normalized TED sensor signal at various measurement points, oriented into machine $\mathrm{X}$ and $\mathrm{Y}$ axes. (c) Topological plot of surface function fit.....

Figure 4: Spatial response function and calculated effective area $\left(\mathrm{A}_{\mathrm{eff}}\right)$ compared to aperture area, cropped to areas of different aperture diameters.

Figure 5: Miniature commercial off-the-shelf (COTS) calibration blackbody placed in the build well of the LPBF machine.

Figure 6: Example comparison of calibration points at different aperture sizes, (a) Signal vs. blackbody temperature. (b) Signal divided by true aperture area. (c) Signal divided by effective aperture areas calculated in Figure 4.

Figure 7: Calibration curve fit to establish $M\left(T_{b b}\right)$ using the inverse Sakuma-Hattori equation [5].

Figure 8: Equivalent source temperature as a function of TED detector signal, plotted at different source diameters and uniform emissivity values. The $10 \mathrm{~mm}, \epsilon=1$ curve at the bottom is the same as in Figure 7.

Figure 9: Finite-element simulation of melt pool surface temperature with potential equivalent source areas $\left(A_{m p}\right)$. Simulation data is provided by the authors of Kollmansburger et al. [6]. Simulation is on nickel alloy 625, $195 \mathrm{~W}$ laser power, $800 \mathrm{~mm} / \mathrm{s}$ scan speed, and $100 \mu \mathrm{m}$ D4 $\sigma$ laser spot diameter, with melt pool length and depth tuned to match those from the 2018 NIST AM Benchmark Test Series [7].......... 15 


\section{Introduction}

In-situ process monitoring in additive manufacturing (AM) utilizes a range of sensor systems and analysis techniques to characterize the quality of the AM parts while they are being fabricated rather than rely on costly or destructive ex-situ qualification. One such technique common in commercial laser powder bed fusion (LPBF) AM systems is coaxial melt pool monitoring. Here, various single-point or imager-based photosensitive detectors are optically aligned with the laser. This allows the sensors to continually observe the radiant emission from the laser-induced melt pool, plume, or other hot incandescing objects as the laser scans throughout each layer during the 3D build.

One such commercial system is the PrintRite3 ${ }^{1}$ system from Sigma Labs, Inc., which is a third-party-integrated, co-axial melt pool monitoring system. It consists of three photodetectors, co-axially aligned with the laser shown in Figure 1. These include associated data acquisition and software processing tools installed on a variety of commercial LPBF systems. PrintRite3D and other commercial co-axial monitoring systems process the sensor signals into various metrics that aim to sense the locally varying melt pool size and/or temperature. Melt pool size and temperature are known to correlate to various defect formation mechanisms or microstructural heterogeneity. For the PrintRite3D, these are called In-Processes Quality Metrics (IPQM $\left.{ }^{\mathrm{TM}}\right)$ and include Thermal Energy Density $\left(\mathrm{TED}^{\mathrm{TM}}\right.$ ) or Thermal Emission Planck (TEP ${ }^{\mathrm{TM}}$ ) metrics. However, while these commercial co-axial melt pool monitoring systems aim to ascribe their sensor signals directly or indirectly to melt pool temperatures, a practical method for thermal calibration has not been identified that can robustly tie these signatures to physical temperature values.

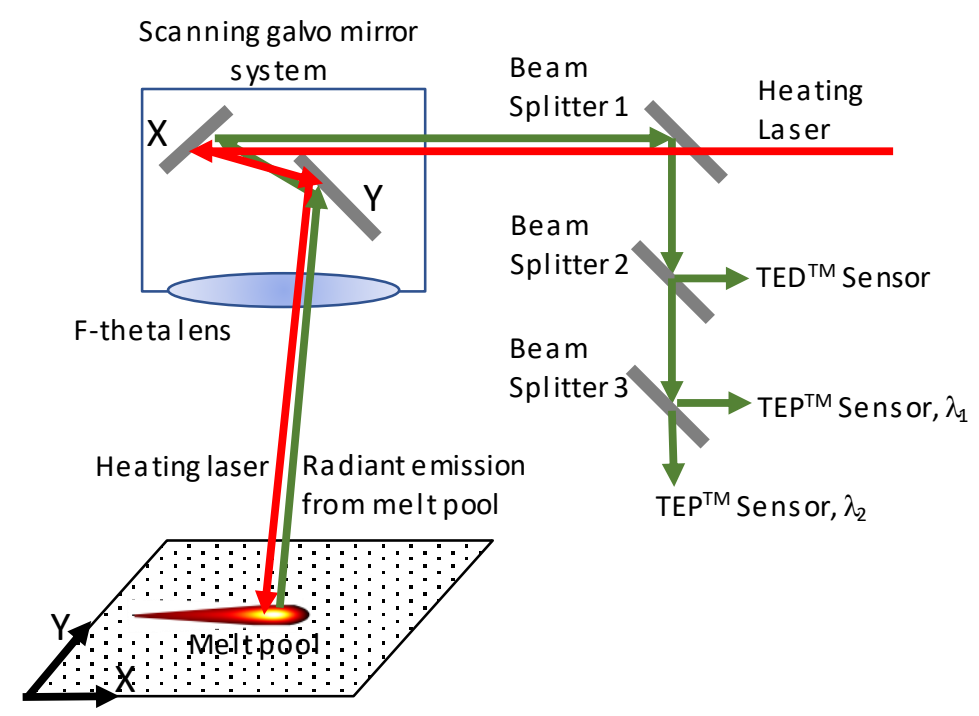

Figure 1: Schematic of optical layout of PrintRite3D co-axial melt pool monitoring system showing TEPTM \& TED $^{\mathrm{TM}}$ sensors.

${ }^{1}$ Certain commercial equipment, instruments, or materials are identified in this paper in order to specify the experimental procedure adequately. Such identification is not intended to imply recommendation or endorsement by the National Institute of Standards and Technology, nor is it intended to imply that the materials or equipment identified are necessarily the best available for the purpose. 
Thermal calibration of radiometric temperature sensors is the process of mapping acquired sensor signals to a radiant temperature of a hypothetical perfect blackbody emitter. In the context of this paper, the hypothetical blackbody emitter is realized by a calibration blackbody, which is designed to output a radiant emission that sufficiently replicates the radiance at specific wavelengths and temperatures dictated by Planck's law [1]. Two primary advantages of a thermal calibration include:

1) A stable source can provide equivalency testing of the sensor(s) for quality assurance, repeatability, tuning, etc.

2) A calibration source can provide physical connection to the thermodynamic temperature of the measurement object.

While \#1 is valuable in and of itself and doesn't necessarily need a Planckian blackbody emitter, \#2 provides a step towards absolute measurement, that is, the assignment of a real thermodynamic temperature to the sensor signal. Thermal calibration itself requires a relatively well-controlled and characterized setup and environment, as well as very stringent assumptions to interpret the calibration uncertainty [2]. Beyond this, enabling a sufficiently accurate absolute temperature measurement using non-contact thermometry in the actual measurement environment requires much greater and numerous assumptions $[3,4]$.

A physical calibration source would preferably match the size and radiant characteristics of the object to be measured. However, this poses some significant challenges when the measurement object is a LPBF melt pool, which is on the order of 100's of micrometers in size scale, and exhibit surface temperature that can range from near ambient to the boiling point of the metal. Potential thermal calibration sources can be divided into two categories: broadband and narrowband, depending on the relative range of wavelengths over which they emit. Broadband sources, such as calibration blackbodies or incandescent sources, provide a Planckian or near Planckian spectral emission over a broad range of wavelengths, typically much over $100 \mathrm{~nm}$. These sources emit at wavelengths within and outside the bandpass of the detector to be calibrated, therefore a lot of energy emitted at those outside wavelengths is wasted. To achieve peak radiance temperatures equivalent to those reached in a LPBF melt pool (greater than $3000{ }^{\circ} \mathrm{C}$ ) at the same size (hundreds of micrometers) requires very high operating power due to the wasted emission outside the waveband or field of view (FoV) of the detector. Narrowband sources, such as high-power light-emitting diodes (LEDs), lasers, or spectral lamps based on the line emission of specific elemental gases or metal vapor, emit over a short region of wavelengths (typically much less than $100 \mathrm{~nm}$ ) and require much lower operating power. However, assigning an equivalent blackbody temperature requires complete knowledge of both source and detector spectral characteristics, particularly if spectral sensitivity bandwidth of the sensor is wider than the spectral output of the narrowband emitter. Additionally, temperature control and current stabilization may be necessary, and/or additional optical integration (e.g., integrating sphere) or other components may be necessary to enable spatial uniformity. Commercial off-the-shelf (COTS) calibration instruments that match the expected radiant characteristics of a LPBF melt pool are not readily available without additional design or fabrication. Calibration blackbodies that reach these temperatures are not appropriately sized for in-situ calibration within an LPBF system. 
However, certain characteristics of the PrintRite3D sensors and their integration into an LPBF system, shown in Figure 1, enable a method for thermal calibration using a miniature COTS calibration blackbody. This method, described in the next section, takes advantage of the fact that the FoV of the detectors at the build plane in the LPBF system is larger than the scale of a LPBF melt pool (typically on the order of hundreds of micrometers). Therefore, a radiance temperature can be mapped to the individual sensor's signals from a COTS calibration blackbody source that is much larger than a melt pool, but also much lower temperature. Radiance temperature, sometimes called apparent temperature, is the equivalent measured temperature if the source had a hypothetical emissivity of $\epsilon=1$. In turn, that signal to radiance temperature mapping can be inverted, such that for a given sensor signal, an 'equivalent source' temperature and size can be calculated. For the PrintRite3D system, this paper shows that the 'equivalent source' temperature and size, obtained from a COTS calibration blackbody, can be on the same order as that expected of an LPBF melt pool.

\section{2. 'Equivalent Source' Theory}

To derive the mathematical basis for the equivalent source method, we start with the measurement equation for an optical detector observing a blackbody source in Eqn. (1) [1]. This equation assumes the detector is linear, meaning the signal output is linearly related to the incident radiant flux on the detector, which is true for most photodetectors over many decibels of dynamic range. Here, $S$ is the sensor signal, typically in units [V] for a preamplified photodetector. $F\left(T_{b b}\right)$ is a functional mapping between the blackbody temperature $\left(T_{b b}\right.$ in $\left.\left[{ }^{\circ} \mathrm{C}\right]\right)$ and the sensor signal $S$, given all other variables are constant. This function $F$ replicates the spectrally-integrated Planckian radiance function $L\left(\lambda, T_{b b}\right)$ [1]. For a source equal to or larger than the FoV, sensor response corresponds to the average intensity of the source across the FoV. However, a relatively small source, much smaller than the FoV, may not elicit the same signal response if positioned at different locations within the sensor FoV. Therefore, the size of the source relative to FoV and its location in FoV (if smaller than FoV) must be considered when calibrating the sensors using different size sources. For this effect, a normalized sensor response term $S_{n}(x, y)$ (unitless) is introduced, representing the normalized signal as a function of $x, y$ position of the source within the FoV. This is especially important since we intend to calibrate using a large source, but infer the temperature of a small, melt pool-size source.

$$
S=F\left(T_{b b}\right)=\alpha \iiint_{\lambda} w(\lambda) S_{n}(x, y) \epsilon_{b b} L\left(\lambda, T_{b b}\right) d A d \lambda
$$

Other terms included within the integral include an assumed non-unity emissivity $\epsilon_{b b}$ of the blackbody and the normalized system spectral response $w(\lambda)$. This includes the spectral response of the detector as well as spectral bandpass of all optics between the sensor and the measurement target surface (i.e., the build plane in the LPBF system). The calibration blackbody emissivity, $\epsilon_{b b}$, is assumed to be spatially uniform and spectrally independent, meaning it can be pulled out of the integrals. Finally, other unchanging variables, such as the angular subtense of the optical system defined by its numerical aperture, attenuation by transmissive or reflective optics, and the gain of the detector, are combined into the linear constant $\alpha$. 
Two parts of the right-hand side of Eqn. (1) can be separated into their respective spatial and spectral counterparts, as shown in Eqn. (2) and Eqn. (3). In Eqn. (2), $A_{\text {eff }}$ is the 'effective area' of the source. If the detector responsivity is spatially uniform across the FoV, then $A_{\text {eff }}$ is the area of the blackbody aperture. Otherwise, $A_{\text {eff }}$ is some value smaller than the blackbody aperture, scaled by the relative nonuniformity of the sensor spatial response $S_{n}(x, y)$. Both measurement of $S_{n}(x, y)$ and calculation of $A_{\text {eff }}$ are shown in the next section. Eqn. (3) simplifies the spectrally integrated Planck's function and sensor responsivity to give another function $M$ that is solely a function of temperature:

$$
\begin{gathered}
\iint_{A} S_{n}(x, y) d A=A_{\text {eff }} \\
\int_{\lambda} w(\lambda) L\left(\lambda, T_{b b}\right) d \lambda=M\left(T_{b b}\right)
\end{gathered}
$$

Finally, the measurement equation in Eqn. (1) can be re-written based on the simplification in Eqn. (2) and Eqn. (3):

$$
S=\alpha \epsilon_{b b} A_{b b, e f f} M\left(T_{b b}\right)
$$

Eqn. (4) can then be used to relate the temperature of a calibration blackbody, $T_{b b}$, to the detector signal $S$, given the other variables are known or assigned.

The crux of the equivalent source method then assumes that there can be some other source measured by the same sensor apparatus with the same linearity constant $\alpha$, but with different parameters $\epsilon, A_{\text {eff }}$, or $T$, that will give the same sensor signal $S$. That hypothetical source could be the approximate size $A_{m p}$, singular temperature, $T_{m p}$, and effective emissivity $\epsilon_{m p}$, of a LPBF melt pool. The sensor signal obtained from that source could then be equated to Eqn. (4) from the blackbody calibration, as shown in Eqn. (5):

$$
\epsilon_{b b} A_{b b, e f f} M\left(T_{b b}\right)=\epsilon_{m p} A_{m p} M\left(T_{m p}\right)
$$


To test and demonstrate this concept, a sensor system is modelled based on realistic parameters. First, we assume calibration blackbody emissivity $\epsilon_{b b}=1$, as well as the hypothetical source $\epsilon_{m p}=1$. Sensor spectral responsivity, $\mathrm{w}(\lambda)$, is assumed to be defined by a narrow waveband of $(580 \pm 5) \mathrm{nm}$ with uniform response within the band. Spatial responsivity of the system, $S_{n}(x, y)$, is presumed to be uniform, such that $A_{e f f, b b}=A_{b b}$. The spectrally integrated radiance function, $M\left(T_{b b}\right)$, is calculated using Planck's law and the spectral responsivity, $w(\lambda)$, according to Eqn. (3) for a range of $T_{b b}=500{ }^{\circ} \mathrm{C}$ to $1200{ }^{\circ} \mathrm{C}$. Note that Wien's approximation to Planck's law could also have been used with minimal loss in accuracy instead of the integral in Eqn. (3), given the narrow bandwidth and central wavelength of $w(\lambda)$ and temperature range of $T_{b b}[1,3]$. Section 4 will show that this function is also evaluated through a physical calibration and curve regression rather than calculated model. By using Planck's law, Eqn. (3) results in the radiance of the source, in units of $\left[\mathrm{W} /\left(\mathrm{m}^{2} \cdot \mathrm{sr}\right)\right]$. Rather than measuring or estimating the angular subtense of the system, which is incorporated into the linear factor $\alpha$, we calculate the surface radiant exitance over a hemisphere $(\Omega=2 \pi \mathrm{sr})$. Multiplying by the source area, $\mathrm{A}_{\mathrm{bb}}$ or $\mathrm{A}_{\mathrm{mp}}$, results in the temperature dependent surface radiant flux in units [W]. The equivalent smaller 'melt pool' temperature is then calculated by rearranging Eqn. (5) such that $T_{m p}=M^{-1}\left[A_{b b} / A_{m p} \cdot M\left(T_{b b}\right)\right]$.

This is calculated for two hypothetical circular sources of diameter $10 \mathrm{~mm}$ and $200 \mu \mathrm{m}$ and plotted in Figure 2. From this plot, it can be seen that the surface radiant flux, which the detector signal is proportional to, from a calibration blackbody with relatively low temperature of $1200{ }^{\circ} \mathrm{C}$ and relatively large aperture diameter of $10 \mathrm{~mm}$, can equate to an equivalent blackbody source that is $2282^{\circ} \mathrm{C}$ and $200 \mu \mathrm{m}$ in diameter.

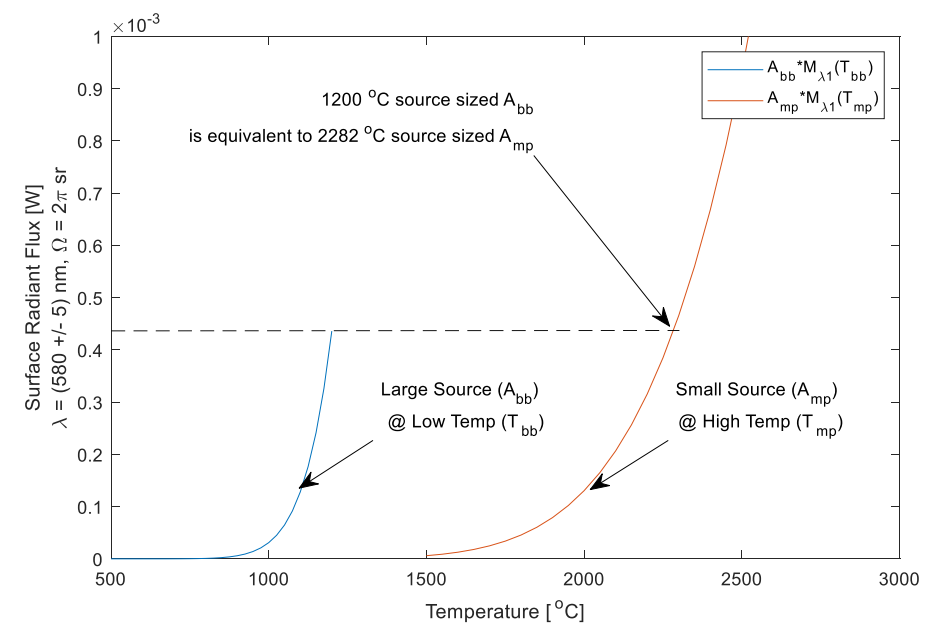

A $10 \mathrm{~mm}$ diameter blackbody at $1200{ }^{\circ} \mathrm{C}$ emits same radiant flux...

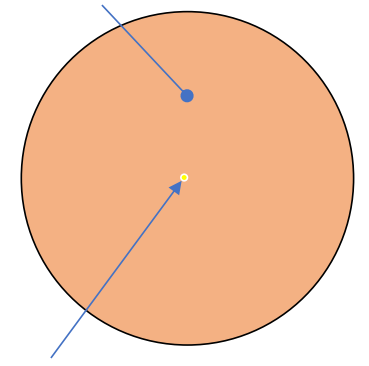

as a $200 \mu \mathrm{m}$ blackbody at $2282{ }^{\circ} \mathrm{C}$ (for the given spectral band)

Figure 2: Left. Calculated temperature-dependent radiant flux from two sources at $10 \mathrm{~mm}$ diameter $\left(A_{b b}\right)$ and $200 \mu \mathrm{m}$ diameter $\left(A_{m p}\right)$. Right: Graphical depiction of the relative areas of a $10 \mathrm{~mm}$ and $200 \mu \mathrm{m}$ diameter source.

It should be noted that the TED detector on the PrintRite3D system does not have a narrow band spectral responsivity centered at $580 \mathrm{~nm}$, as was used to demonstrate the concept in Figure 2, but a response covering a broader waveband. The spectral response of the TED detector is defined by transmission of the f-theta lens, spectrally-variant 
reflectance of the galvo mirrors and beam splitter 1 in Figure 1, and any other transmissive or reflective components in the optical path. While the actual spectral response is not measured here, the conceptual calculation in Figure 2 was repeated using hypothetical full spectral response of a typical silicon-based photodetector $(\mathrm{w}(\lambda)$ in Equation (3)), with peak responsivity at approximately $980 \mathrm{~nm}$. For the equivalent radiant flux of a $10 \mathrm{~mm}$ circular source at $1200{ }^{\circ} \mathrm{C}$, a $200 \mu \mathrm{m}$ source would equate to $3811^{\circ} \mathrm{C}$. This shows that for wider spectral response of the detector, the calculated equivalent 'small source' temperature increases.

\section{Measurement Setup}

While the previous example showed that it is feasible to calculate an 'equivalent source' temperature from a lower temperature calibration source, it assumed that this spatial response of the detector $S_{n}(x, y)$ is uniform. For the PrintRite3D system, that is not the case, so we directly measured the spatial response of the detector at the build plane of a LPBF machine. This was done by putting a small, mirrored precision aperture $(100 \mu \mathrm{m}$ diameter), back-lit by fiber-coupled tungsten halogen light source within the LPBF machine build chamber. The source was mounted to two motorized micrometer stages mounted at $90^{\circ}$. These were then mounted to the build platform of the LPBF machine, shown in Figure 3a. Thich was raised until the aperture surface was approximately at the level of the LPBF recoater blade. Due to the size of the micrometer stages, they had to be rotated to fit within the LPBF build well. This rotation angle was measured using a protractor, so micrometer stage positions could be mapped to the LPBF machine nominal $\mathrm{X}$ and $\mathrm{Y}$ directions. The motorized micrometer stages were then positioned at various points throughout the build chamber, shown in Figure $3 \mathrm{~b}$. The signal from the TED detector on the PrintRite3D system was then measured at each aperture position using a precision multimeter (resolution $10 \mu \mathrm{V}$ ). Detector gain was set to $30 \mathrm{~dB}$. The detector noise floor or zero level $\left(S_{0}\right)$ was measured by covering the f-theta lens in the LPBF system with a sheet of paper. All detector signal values are expressed with zero-level noise subtracted $\left(S-S_{0}\right)$.

Apart from measuring the detector spatial response, the aperture measurement also identified the location of peak sensitivity, likely aligned with the LPBF system laser while it was held stationary during the tests. The aperture was positioned at this maximum sensitivity location. Its location was measured using digital calipers, referenced from the inside edges of the build well. This location was noted for later positioning and alignment of the calibration blackbody. 

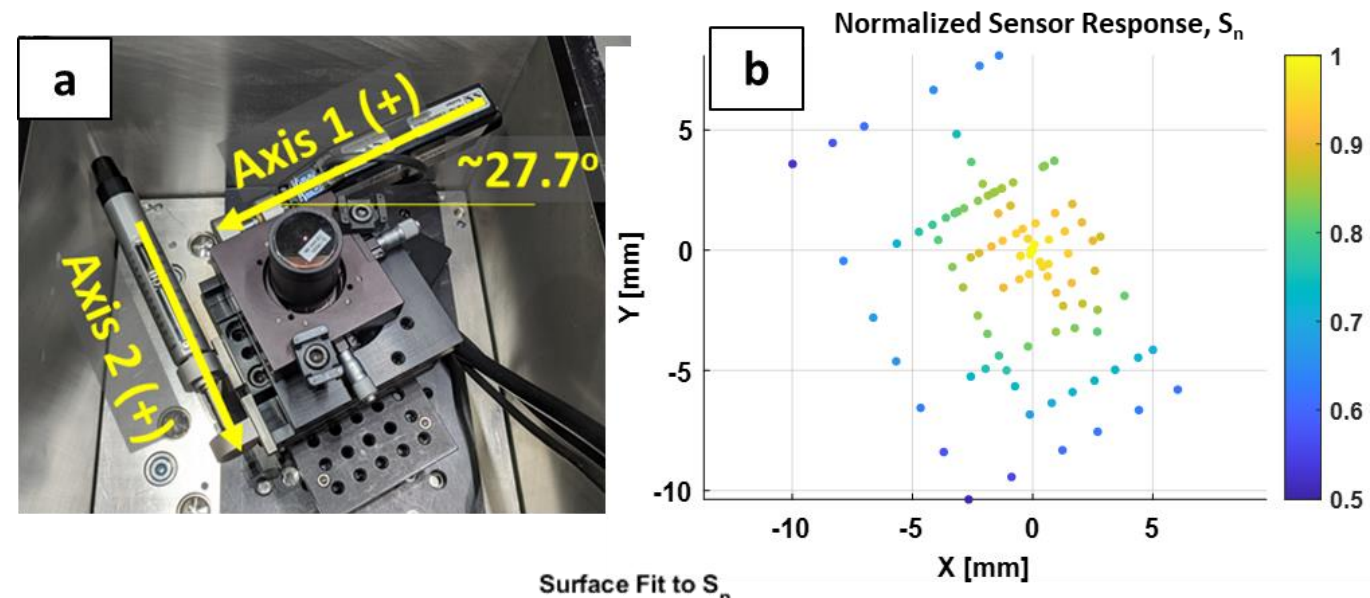

Surface Fit to $S_{n}$

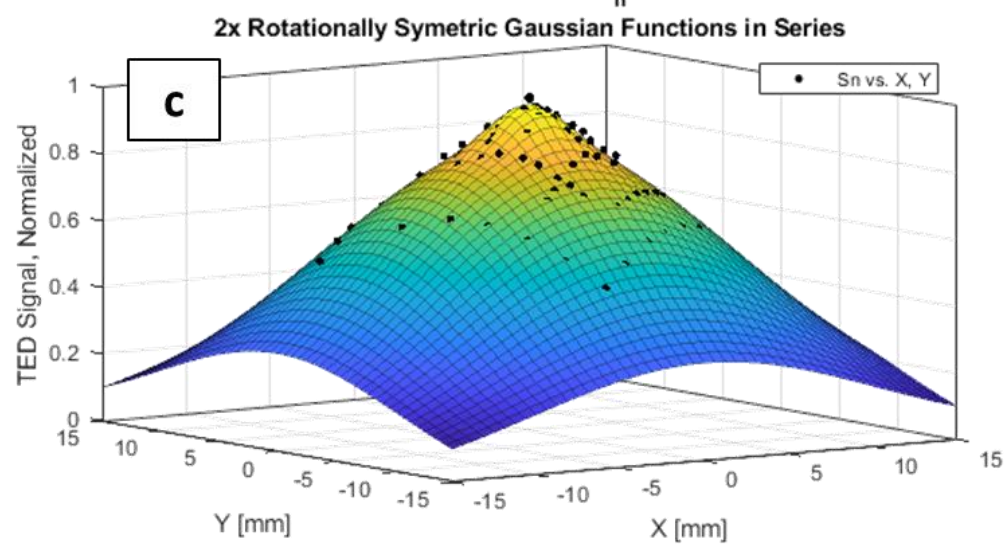

Figure 3: Measurement of the sensor spatial response function $S_{n}(x, y)$. (a) Experiment setup, consisting of a mirrored aperture ( $100 \mu \mathrm{m}$ diameter), back-lit by fiber light and mounted on orthogonal micrometer stages. (b) Normalized TED sensor signal at various measurement points, oriented into machine $\mathrm{X}$ and $\mathrm{Y}$ axes. (c) Topological plot of surface function fit.

The measured sensor signals were then normalized vs. the peak signal value. Various analytical $2 \mathrm{D}$ surface functions were then tested using linear regression analysis. While a 2D conical function resulted in best fit $\left(\mathrm{r}^{2}=0.9951, \mathrm{RMSE}=0.0088\right)$, extrapolation of points outside the measured $\mathrm{X}$ and $\mathrm{Y}$ range would have resulted in negative values, which are physically unrealistic. Instead, two rotationally symmetric Gaussian functions added in series, requiring four fit coefficients, resulted in $\mathrm{r}^{2}=0.9915$ and root-mean squared error $(\mathrm{RMSE})=0.0115$, and is shown in Figure 3c. While this surface function does not intersect $S_{n}=0$, which can be used to define the field of view of the detector, $S_{n}=0.05$ occurs at a diameter of $48.7 \mathrm{~mm}$.

This continuous surface function, shown again in Figure 4a, was then restricted over finite circular domains in X and Y representing three apertures, shown in Figure 4b-c. Using Eqn. (2), the effective areas were calculated and compared to the actual aperture areas, shown in the titles of each subfigure in Figure $4 \mathrm{~b}-\mathrm{c}$. 

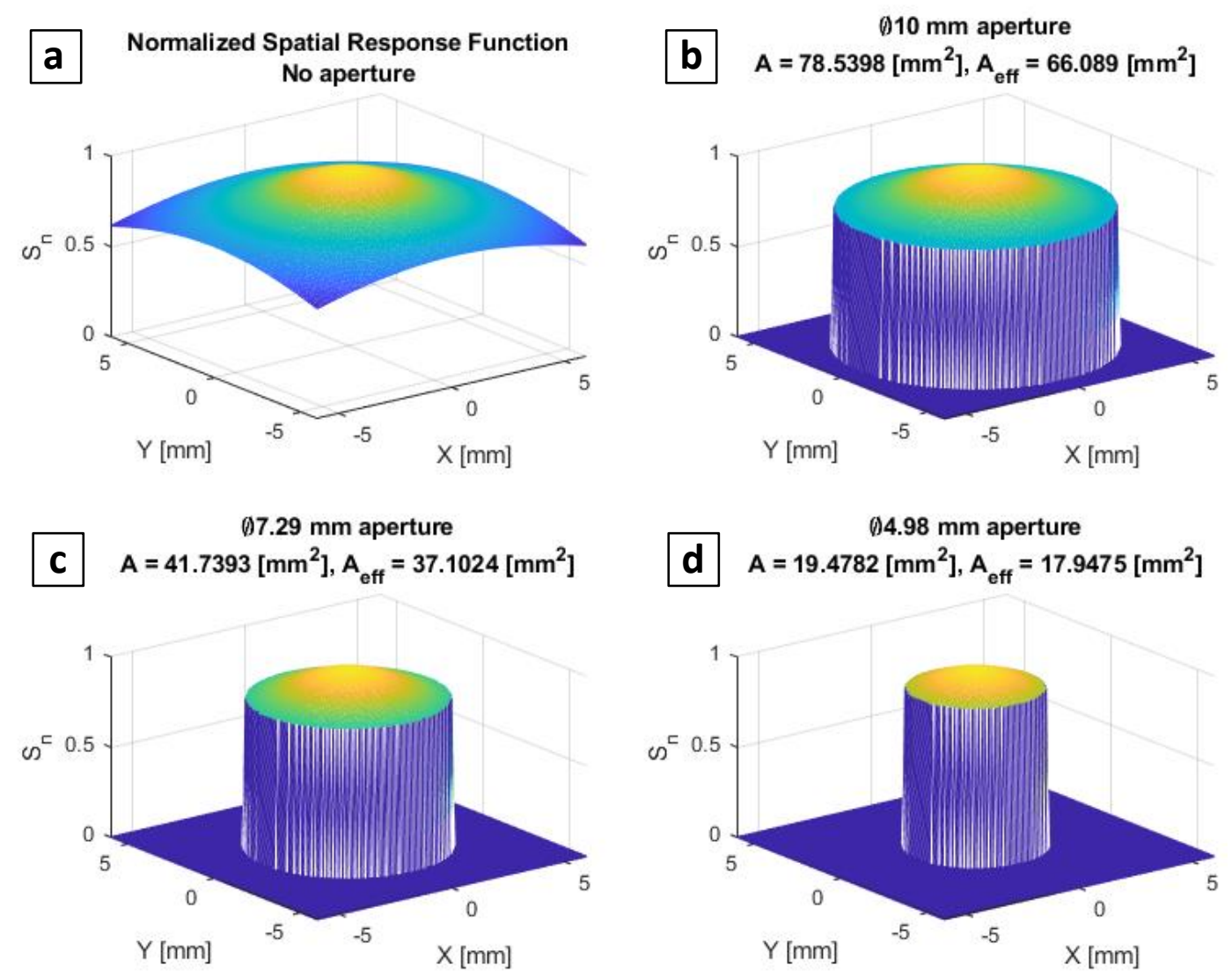

Figure 4: Spatial response function and calculated effective area ( $\left.\mathrm{A}_{\mathrm{eff}}\right)$ compared to aperture area, cropped to areas of different aperture diameters.

A COTS miniature blackbody was then mounted to the LPBF system build platform. It was centered based on the measured peak spatial responsivity in Figure 3, and positioned in $\mathrm{Z}$ such that the opening aperture is level with the LPBF machine's recoater blade. This miniature calibration blackbody, shown in Figure 5, has a cylindrical cavity with conical bottom, opening aperture of $10 \mathrm{~mm}$, and a vendor-stated temperature range of $50{ }^{\circ} \mathrm{C}$ to $1200{ }^{\circ} \mathrm{C}$ and stated accuracy of $0.2^{\circ} \mathrm{C}$. Three apertures, consisting of standard metal washers with internal diameters equal to that in Figure $4 \mathrm{~b}-\mathrm{c}$, could be placed and centered over the calibration blackbody opening aperture. 


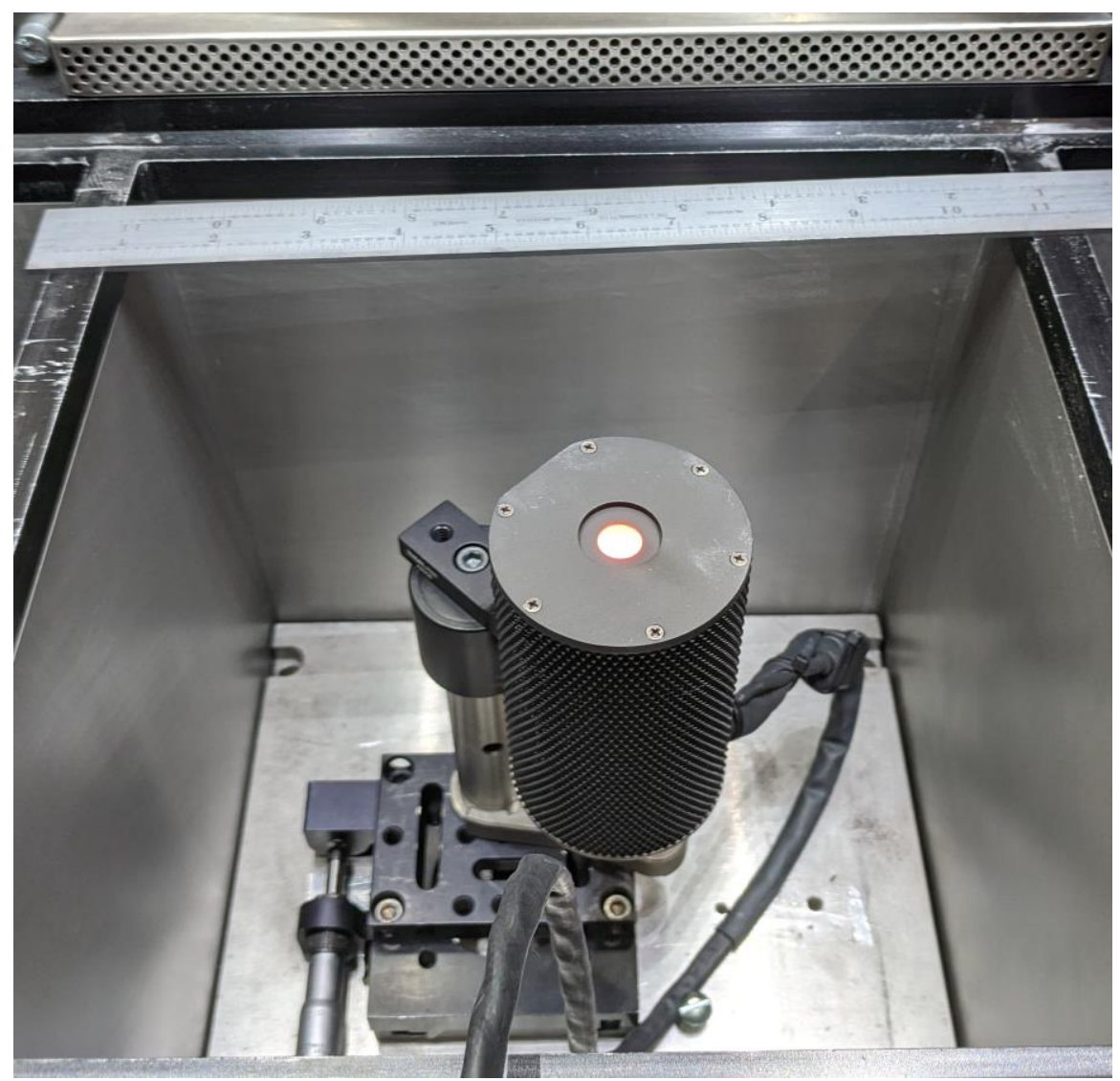

Figure 5: Miniature commercial off-the-shelf (COTS) calibration blackbody placed in the build well of the LPBF machine.

The PrintRite3D system's TED detector was set to $30 \mathrm{~dB}$ gain, and signal again monitored with the precision multimeter while the calibration blackbody setpoint temperature was varied from $700{ }^{\circ} \mathrm{C}$ to $1200{ }^{\circ} \mathrm{C}$, with adequate time for the blackbody to stabilize within $1{ }^{\circ} \mathrm{C}$ of each setpoint. At several intermittent setpoints, the TED detector gain was set to $30 \mathrm{~dB}$ or $50 \mathrm{~dB}$ to determine linearity between gain settings and the three different aperture sizes to evaluate the calculation of $A_{\text {eff. }}$.

It should be noted that there is a known spatial nonuniformity of the calibration blackbody radiance, which incurs a slight dip in radiance in the center equating to approximately $10{ }^{\circ} \mathrm{C}$ difference (measured at $1200{ }^{\circ} \mathrm{C}$ over a $(850 \pm 20) \mathrm{nm}$ waveband). This may contribute to the total measurement uncertainty in an absolute radiance or temperature calibration. However, this likely has much less effect than the spatial response shown in Figure 3c, and since the blackbody uniformity dips in the center, this would reduce the effect stemming from the central peak in Figure $3 \mathrm{c}$.

\section{Calibration Results}

According to Eqn. (4), the measured signal for the same detector should scale linearly with the aperture area $A_{b b}$. If the spatial responsivity is not uniform over the field of view, we hypothesized in the previous section that an effective aperture area, $A_{b b, \text { eff }}$ could be calculated according to Eqn. (3). This would provide a more linear relationship with 
the detector signal than $A_{b b}$. Figure 6a shows the measured detector signal minus zero level, $S-S_{0}[\mathrm{mV}]$, at several select blackbody setpoint temperatures $T_{b b}$. This demonstrates how the detector signal increases with aperture size. To test that the signal increases linearly with aperture size, and to see if $A_{b b, e f f}$ provides a better linearization, Figure $6 b$ and $6 c$ compare the signal values scaled by the true aperture area, $A_{b b}$, and the effective aperture areas $A_{b b, e f f}$ calculated in Figure 4. This should cause each curve to overlap, with an improved overlap using $A_{b b e f f}$ in Figure $6 \mathrm{c}$. However, it can be seen in Figure $6 \mathrm{~b}$ that this is not the case for $A_{b b}$, and scaling by $A_{b b, e f f}$ improves the curve overlap only moderately in Figure 6c.
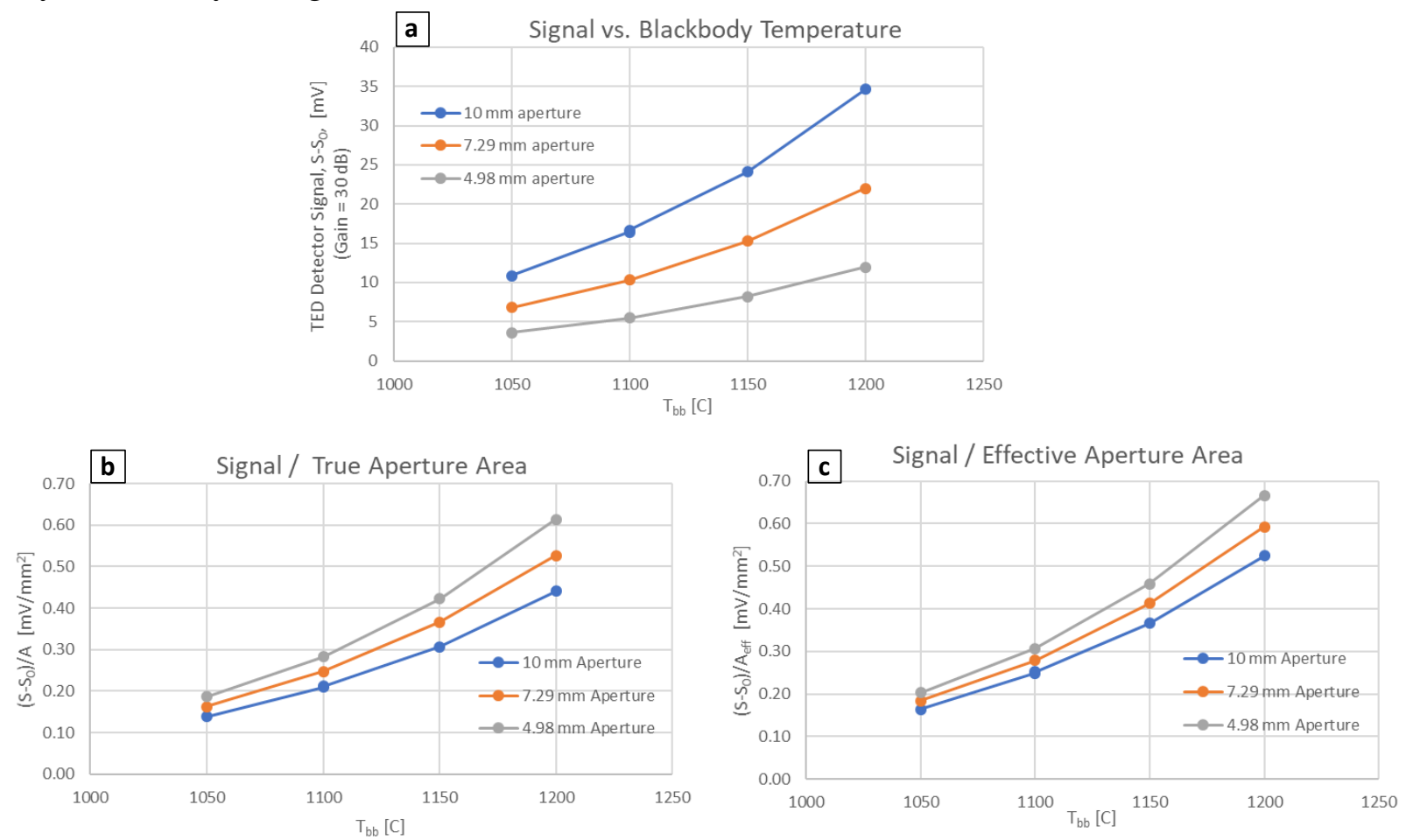

Figure 6: Example comparison of calibration points at different aperture sizes, (a) Signal vs. blackbody temperature. (b) Signal divided by true aperture area. (c) Signal divided by effective aperture areas calculated in Figure 4.

Several reasons for this non-linearity with aperture area are hypothesized and discussed at the end of this document. Nevertheless, we proceed with evaluating the calibration results from the $10 \mathrm{~mm}$ diameter aperture blackbody measurements.

First, we evaluate the function $M$ used in Eqns. (3) to (5) by non-linear regression between the blackbody temperature $T_{b b}$ and zero-subtracted detector signal $S$, using the inverse Sakuma-Hattori (SH) equation given in Eqn. (6) [5]. A,B, and C are fit coefficients, and $\mathrm{c}_{2}=14388 \mu \mathrm{m} \cdot \mathrm{K}$ is the second radiation constant. The $\mathrm{SH}$ equation, which maps blackbody temperature to sensor signal in its forward form given in Eqn. (7), is often used in radiation thermometry as an invertible analytical approximation to the spectrally-integrated Planck radiance function in Eqn. (3) [2, 3]. A plot of the regression results with $95 \%$ confidence intervals is shown in Figure 7 . Resulting fit coefficients were $\mathrm{A}=0.6408, \mathrm{~B}=362.4$, and $\mathrm{C}=1.171 \times 10^{7}\left(\mathrm{r}^{2}=0.9991, \mathrm{RMSE}=4.934{ }^{\circ} \mathrm{C}\right)$. Though 
typically done with temperature units $[\mathrm{K}]$, the fit was done in units $\left[{ }^{\circ} \mathrm{C}\right]$, with fit results deemed sufficiently accurate.

$$
\begin{gathered}
T=M^{-1}(S)=\frac{c_{2}}{A \ln \left(\frac{C}{S}+1\right)}-\frac{B}{A} \\
S=M(T)=\frac{C}{\exp \left(\frac{C_{2}}{A T+B}\right)}
\end{gathered}
$$

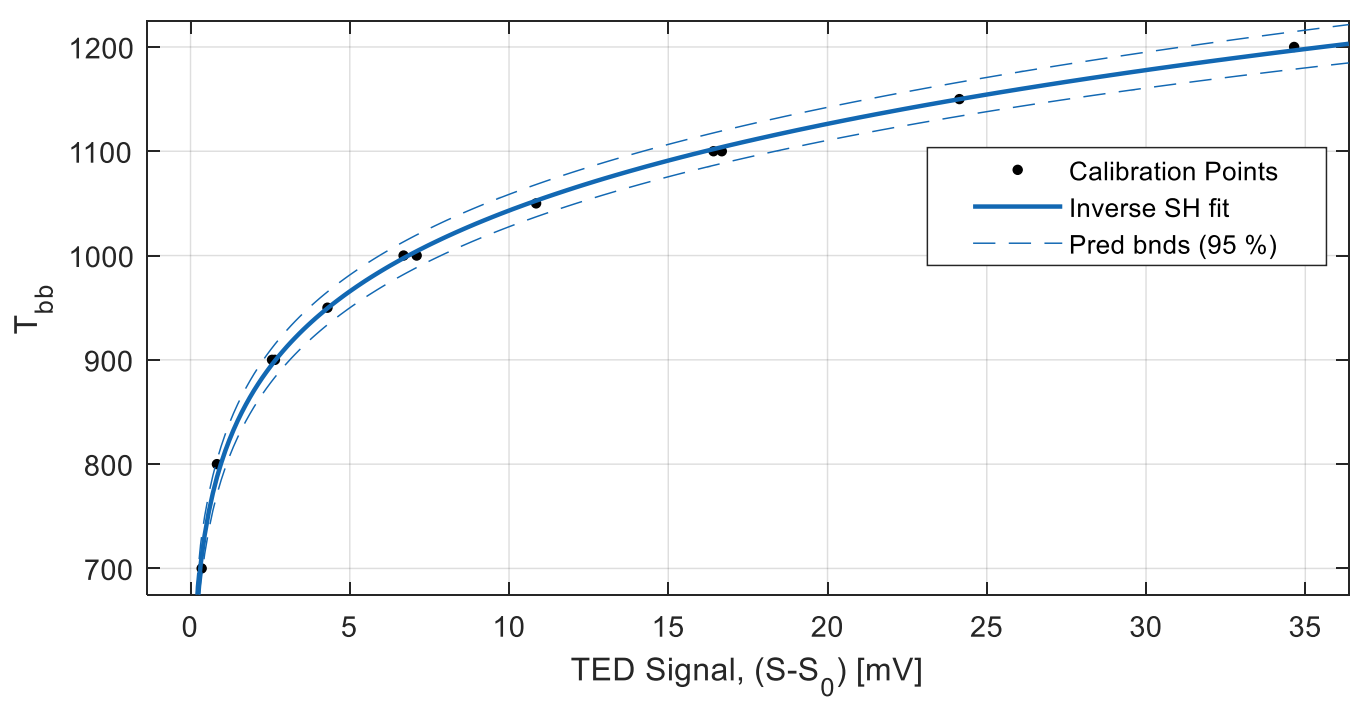

Figure 7: Calibration curve fit to establish $M\left(T_{b b}\right)$ using the inverse Sakuma-Hattori equation [5].

Finally, to demonstrate the concept of the equivalent source method, Eqn. (5) is rearranged into Eqn. (8), which enables calculation of a hypothetical, melt pool-scale source of uniform temperature $T_{m p}$. Example temperature curves are calculated for three circular sources of diameter $100 \mu \mathrm{m}, 200 \mu \mathrm{m}$, and $300 \mu \mathrm{m}$ (to formulate $A_{m p}$ ), and three emissivity values of $\epsilon=1,0.5$, and 0.2 , with results shown in Figure 8 . This uses the $\epsilon_{b b}=1$, and $A_{b b, e f f}=66.09 \mathrm{~mm}^{2}$ for the $10 \mathrm{~mm}$ aperture.

$$
T_{m p}=M^{-1}\left(\frac{\epsilon_{b b} A_{b b, e f f}}{\epsilon_{m p} A_{m p}} M\left(T_{b b}\right)\right)
$$

As previously mentioned, this does not enable calculation of true surface temperature from a measured detector signal. However, it does enable a statement to be made, such as:

\section{"A measured signal value of $S[\mathrm{mV}]$ is similarly obtained from a circular source of size $A_{m p}\left[\mu m^{2}\right]$ at uniform temperature $T_{m p}\left[{ }^{\circ} \mathrm{C}\right]$ and effective emissivity $\epsilon_{m p}$."}

For example, in Figure 8, a measured signal value of $20 \mathrm{mV}$ is similarly obtained from a circular source of $200 \mu \mathrm{m}$ diameter, emissivity of 0.5 , at a uniform temperature of $3400{ }^{\circ} \mathrm{C}$. While only a step towards absolute temperature measurement, we discuss in 
the next section why these values are within the range of those that might be expected from a LPBF melt pool.

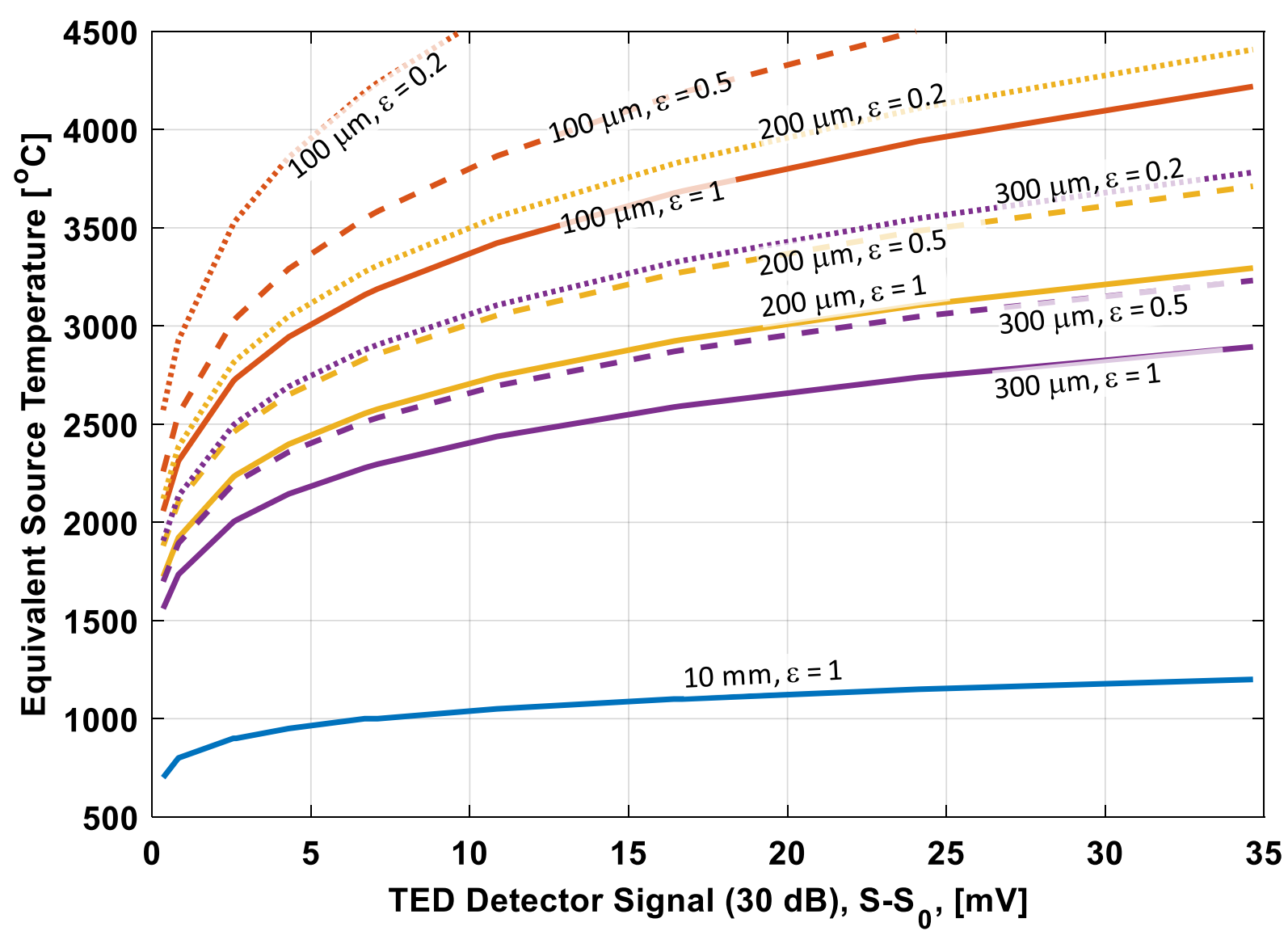

Figure 8: Equivalent source temperature as a function of TED detector signal, plotted at different source diameters and uniform emissivity values. The $10 \mathrm{~mm}, \epsilon=1$ curve at the bottom is the same as in Figure 7.

\section{Discussion}

The response of a co-axial melt pool monitoring sensor will ultimately depend on multiple factors pertaining to the complex physics within the melt pool and surrounding area. For example, individual sources may stem from:

1) The surface area of the melt pool, or the size of the thermal field in and around the melt pool.

2) The temperature of the thermal field in and around the melt pool.

3) The local emissivity, based on local temperature, wavelength, surface roughness and topology, etc.

4) Other incandescing sources within the field of view, such as plume or spatter. 
Identifying the relative contributions of each of these factors to the overall sensor signal or extracting temporal or frequency-domain features that correlate to these factors is an ongoing topic of research. However, we can make use of tuned computational simulations of a LPBF melt pool to demonstrate the feasibility of the size and temperature ranges shown in Figure 8.

Figure 9 shows the top surface temperature results of a finite element simulation of a LPBF melt pool with the different hypothetical source sizes from Figure 8 outlined. Details of the melt pool simulation formulation are provided in [6]. While absolute accuracy of the simulation result is not necessary for this analysis, it provides a glimpse into potential scale of the temperature field in and around the melt pool. The relative size and temperature values within each hypothetical source diameter in Figure 9, are commensurate with those calculated in Figure 8.

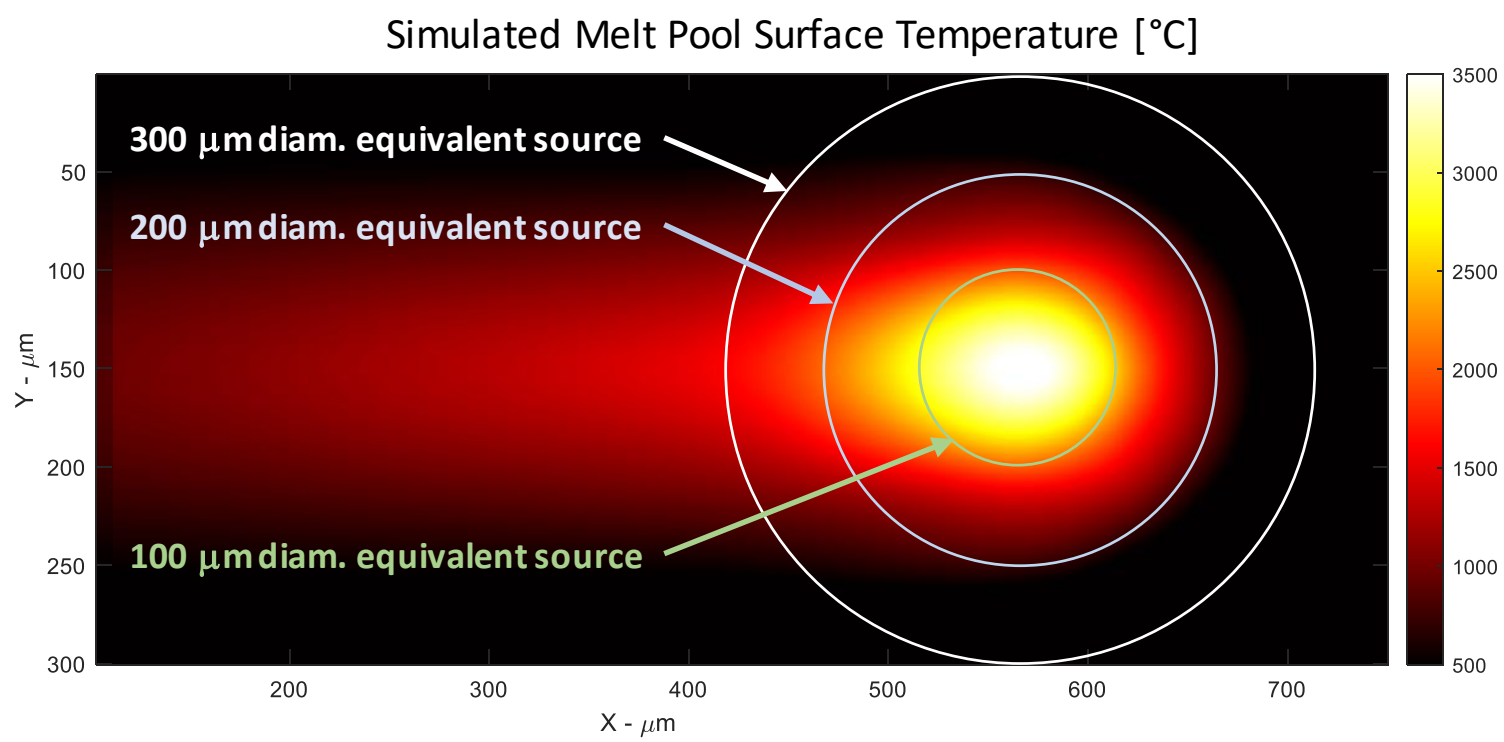

Figure 9: Finite-element simulation of melt pool surface temperature with potential equivalent source areas $\left(A_{m p}\right)$. Simulation data is provided by the authors of Kollmansburger et al. [6]. Simulation is on nickel alloy 625, $195 \mathrm{~W}$ laser power, $800 \mathrm{~mm} / \mathrm{s}$ scan speed, and $100 \mu \mathrm{m}$ D4 $\sigma$ laser spot diameter, with melt pool length and depth tuned to match those from the 2018 NIST AM Benchmark Test Series [7].

\section{Improvements and Future Work}

In Figure 6, we tested the hypothesis that the ratio of two measured signal values should approximately equal the ratio of the two source aperture areas they are obtained from, and this would help to validated the equivalent source method. However, these lines did not overlap, and use of the effective aperture area $A_{\text {eff }}$ based on the measured nonuniform spatial responsivity $S_{n}$ provided only moderate improvement. To make the lines overlap more effectively, the spatial responsivity function $\mathrm{S}_{\mathrm{n}}(\mathrm{x}, \mathrm{y})$ would have to be 'sharper', or have a steeper decline in values from the central to outer regions than that depicted in Figure 3 . That, in effect, would make $A_{\text {eff }}$ for the $10 \mathrm{~mm}$ aperture relatively smaller, and 
that for the $4.98 \mathrm{~mm}$ aperture relatively larger. Several improvements to the measurement setup are identified:

- Use a diffuser on the fiber light, or a more Lambertian source. The fiber may have some angular dependence on its output radiant intensity, which may affect the spatial responsivity measurement.

- Use sharp-edged, precision apertures in front of the calibration blackbody. The apertures used had cylindrical opening cavities, which may introduce spurious reflections.

- Ensure more precise positioning and alignment of the blackbody with the center of the peak spatial responsivity $S_{n}(x, y)$.

- Ensure more precise positioning and centering of the apertures over the blackbody and the peak spatial responsivity.

- Re-measure the spatial responsivity with a series of micro-apertures (e.g., larger and smaller than the $100 \mu \mathrm{m}$ used here) to determine any effect due to the aperture size.

Future work will entail repeating the calibration procedures outlined in this document, with improvements, for the TED and both TEP sensors on the PrintRite3D system. Further studies will then utilize these calibrated sensors in actual in-situ measurements in the LPBF system. These may include simplified process mapping (single track or single layer) tests and 3D builds, with the goal of building a more robust physical connection between sensor signatures, and the real thermal conditions of the LPBF melt pool.

\section{Conclusions}

This paper outlined a new conceptual method for thermally calibrating co-axially aligned melt pool monitoring photodetectors using a commercial off-the-shelf calibration blackbody. This method, called the 'equivalent source' method, extrapolates a thermal calibration of a low temperature, large aperture area calibration blackbody to that of a small area, high temperature hypothetical source. This is comparable to the size and temperature scale of that expected from an LPBF melt pool.

This method was demonstrated on a commercial, third party integrated melt pool monitoring system on a commercial LPBF machine. The underlying theory behind the method was tested using various aperture sizes on the calibration blackbody. This did not yield expected results based on the theory, but several potential improvements to the measurement setup were given.

Nevertheless, calculation of various hypothetical sources using the equivalent source theory were demonstrated and showed that this extrapolation technique could yield calculated source temperatures above $3000{ }^{\circ} \mathrm{C}$ for source sizes less than $200 \mu \mathrm{m}$. While experiment improvements will be necessary, the method for thermal calibration of melt pool monitoring detectors demonstrated here provides an idea on how the results of such calibration can be communicated and compared to a real LPBF melt pool. 


\section{References}

[1] DeWitt DP, Nutter GD (1988) Theory and practice of radiation thermometry (Wiley Online Library).

[2] Fischer J, Saunders P, Sadli M, Battuello M, Park C, Zundong Y, Yoon H, Li W, van der Ham E, Sakuma F (2008) Uncertainty budgets for calibration of radiation thermometers below the silver point. CCT-WG5 on Radiation Thermometry. (BIPM).

[3] Saunders P (2007) Radiation thermometry: fundamentals and applications in the petrochemical industry (SPIE press), Vol. TT78.

[4] DeWitt DP, Richmond JC (1985) Applications of Radiation Thermometry: A Symposium (ASTM International, Philadelphia, PA).

[5] Sakuma F, Hattori S (1982) Establishing a practical temperature standard by using a narrow-band radiation thermometer with a silicon detector. Temperature: Its Measurement and Control in Science and Industry (AIP, New York), Vol. 5, pp 421-427.

[6] Kollmannsberger S, Carraturo M, Reali A, Auricchio F (2019) Accurate Prediction of Melt Pool Shapes in Laser Powder Bed Fusion by the Non-Linear Temperature Equation Including Phase Changes. Integrating Materials and Manufacturing Innovation 8(2):167-177. https://doi.org/10.1007/s40192-019-00132-9

[7] Lane B, Heigel J, Ricker R, Zhirnov I, Khromschenko V, Weaver J, Phan T, Stoudt M, Mekhontsev S, Levine L (2020) Measurements of melt pool geometry and cooling rates of individual laser traces on IN625 bare plates. Integrating Materials and Manufacturing Innovation. https://doi.org/10.1007/s40192-020-00169-1 